\title{
Infrared image and visible image fusion based on wavelet transform
}

\author{
Zehua Zhou Min Tan \\ Department of Electronics \& Electrical Engineering \\ Hefei University \\ Hefei, China \\ E-mail: zhouzh@hfuu.edu.cn
}

\begin{abstract}
The same scene, the infrared image and visible image fusion can concurrently take advantage of the original image information can overcome the limitations and differences of a single sensor image in terms of geometric, spectral and spatial resolution, to improve the quality of the image, which help to locate, identify and explain the physical phenomena and events. Put forward a kind of image fusion method based on wavelet transform. And for the wavelet decomposition of the frequency domain, respectively, discussed the principles of select high-frequency coefficients and low frequency coefficients, highlight the contours of parts and the weakening of the details section, fusion, image fusion has the characteristics of two or multiple images, more people or the visual characteristics of the machine, the image for further analysis and understanding, detection and identification or tracking of the target image.
\end{abstract}

Keywords- wavelet transform; image fusion; infrared image; visible light images

\section{INTRODUCTION}

Image fusion refers to the integrated two or more source images. The purpose of image fusion is through the extraction and the pieces of image information in order to more accurately target the same scene, a more comprehensive, more reliable description of the image. Image fusion technology research showed a rising trend, the application area throughout the remote sensing image processing, computer vision, automatic target recognition, robotics, medical image processing and other fields. Therefore, image fusion is important.

In nature, all objects can be infrared radiation, thus using the detector determination of the target and background infrared difference between infrared images, the image formed by the thermal infrared is called a heat map. The thermal image of the target and the visible light image of the target, the goal of visible light images, it is not the eye can see, but the target surface temperature distribution of the image, in other words, infrared thermal imaging human eye can not see the target surface temperature distribution into a thermal image of the human eye can see representatives of the target surface temperature distribution, the infrared image on the shape of the target to reflect better the anti-stealth, the identification camouflage and anti-jamming ability, easy imaging. At the same time, due to its transmission rate encountered fog and smoke vision can shoot. However, compared with visible light images, infrared images, the contrast of the object and the background is low, edge blur, noisy, difficult to use conventional edge extraction method to extract the target contour information. Visible images and infrared images have their own advantages and disadvantages, so the formation of two of the same target image data fusion, information fusion of the two complementary, to achieve the purpose of image enhancement amount of information.

Currently the mainstream method of image fusion, airspace integration, high-pass filter integration, integration of the pyramid decomposition of images and wavelet transform fusion method ${ }^{11-7]}$. Wavelet transform the original image can be decomposed into a series of sub-images of the resolution and frequency domain characteristics of different directions, can fully reflect the local variation of the original image, orthogonal wavelet transform in addition to the two adjacent scale image information, in the process of wavelet decomposition, the same amount of image data, while the integration of layers parallel wavelet transform multiresolution structure to solve the image grayscale characteristics to the fusion difficulties.

\section{TWO DIMENSIONAL DISCRETE WAVELET TRANSFORM}

In practical use, in particular, continuous wavelet discretization must be implemented on the computer. Therefore it is necessary to discuss the discretization of continuous wavelet $y_{a, b}(t)$ and continuous wavelet transform $W_{f}(a, b)$.Should be emphasized that this discretization parameters for the continuous scale a parameter and continuous pan b, rather than time t. This is different from our previous habits.For convenience, in the discretization, a can only take positive. Typically, the continuous wavelet transform scale parameter a and translation parameter $\mathrm{b}$ of the discrete formulas were taken as $a=a_{0}^{j}, b=b_{0}^{j}$, here $j \hat{\mathrm{I}} Z$, expansion step $a_{0}{ }^{1} 1$ is a fixed value, for convenience, always assuming $a_{0}>1$.Therefore, the corresponding discrete wavelet function $y_{j, k}(t)$ can be written:

$$
\psi_{j, k}(t)=\frac{1}{\sqrt{\left|a_{0}\right|}} \psi\left(\frac{t-k a_{0}^{j} b_{0}}{a_{o}^{j}}\right)=\frac{1}{\sqrt{\left|a_{0}\right|}} \psi\left(a_{0}^{-j} t-k b_{0}\right)
$$


Discretization of wavelet transform coefficients can be expressed as:

$$
C_{j . k}=\int_{-\infty}^{+\infty} f(t) \psi_{j, k}^{*}(t) d t \leq f, \psi_{j, k}>0
$$

The reconstruction formula is:

$$
f(t)=C \sum_{-\infty}^{\infty} \sum_{-\infty}^{\infty} C_{j, k} \psi_{j, k}(t)
$$

$\mathrm{C}$ is a signal-independent constant. How to choose $a_{0}$ and $b_{0}$, in order to ensure the accuracy of the reconstructed signal? Obviously, the network points should be secret ( $a$ and $b$ as small as possible), because if the network points are more sparse, the use of the wavelet function $y_{j, k}(t)$ and the discrete wavelet coefficients $C_{j, k}$ less signal reconstruction accuracy will be lower. Since images are two-dimensional signal, so you first need to wavelet transform by the one-dimensional to the twodimensional. Assume that $f\left(x_{1}, x_{2}\right)$ is a two-dimensional signal, $x_{1}, x_{2}$, respectively, is the abscissa and ordinate, $y\left(x_{1}, x_{2}\right)$ denotes the two-dimensional wavelet, the corresponding scaling function is $y\left(x_{1}, x_{2}\right)$. If the scaling function can be separated, that is $f\left(x_{1}, x_{2}\right)=j\left(x_{1}\right) * f\left(x_{2}\right)$. Let $y\left(x_{1}\right)$ and $f\left(x_{1}\right)$ correspond to a one-dimensional wavelet function, the twodimensional dyadic wavelet can be expressed for the following three separable orthogonal wavelet basis functions:

$$
\begin{aligned}
& y^{1}\left(x_{1}, x_{2}\right)=j\left(x_{1}\right) y\left(x_{2}\right) \\
& y^{2}\left(x_{1}, x_{2}\right)=y\left(x_{1}\right) j\left(x_{2}\right) \\
& y^{3}\left(x_{1}, x_{2}\right)=y\left(x_{1}\right) y\left(x_{2}\right)
\end{aligned}
$$

This shows that in the case of separable two-dimensional multi-resolution two-step. Its decomposition is shown in Fig. 1.

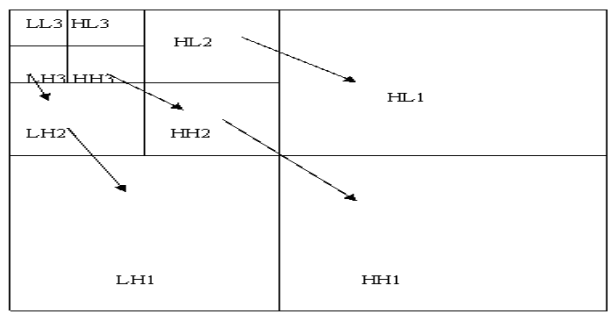

Figure 1. Iimages of the three wavelet decomposition figure
The specific steps along the $x_{1}$ direction, respectively, $f\left(x_{1}\right)$ and $y\left(x_{2}\right)$ do analysis, $f\left(x_{1}, x_{2}\right)$ broken down into two parts of smooth and detail, then do the same analysis of the two parts along the $x_{2}$ direction use $f\left(x_{2}\right)$ and $y\left(x_{1}\right)$. The four outputs from the processing by the $f\left(x_{1}\right)$ and $f\left(x_{2}\right)$ all the way to the first class of smooth approximation $A_{1} f\left(x_{1}, x_{2}\right)$, the other three outputs $D_{1}^{1} f\left(x_{1}, x_{2}\right), \quad D_{1}^{2} f\left(x_{1}, x_{2}\right), \quad D_{1}^{3} f\left(x_{1}, x_{2}\right)$ are the details of the function. If $f\left(x_{1}\right)$ and $y\left(x_{1}\right)$ correspond to spectrum $f(w), y(w)$ conceived as the ideal half-band low-pass filter $h$ and high-pass filter $g, A_{1} f\left(x_{1}, x_{2}\right)$ reflects the low frequency components in both directions of the $x_{1}, x_{2}, D_{1}^{1} f\left(x_{1}, x_{2}\right)$ reflected high-frequency components of the low-frequency component of the horizontal direction and vertical direction, $D_{1}^{2} f\left(x_{1}, x_{2}\right)$ reflected high-frequency components of the horizontal direction and vertical low-frequency component, $D_{1}^{3} f\left(x_{1}, x_{2}\right)$ reflected high-frequency components in both directions. The image wavelet transform is to use low-pass filter is a high-pass filter $b$ of the ranks of the image filtering (convolution), then take one of the next sampling. Such wavelets transform results put the image can be decomposed as a low-frequency sub-band (horizontal and vertical directions after low-pass filter) $L L$ and three high frequency sub-bands, use $H L$ as the horizontal high-pass and vertical low-pass sub-band, use $L H$ as the horizontal low-pass and vertical high-pass sub-band, use $H H$ as the horizontal high-pass and vertical high-pass sub-band. The resolution is $1 / 2$, the frequency range varies.

\section{IMAGE FUSION ALGORITHM BASED ON WAVELET TRANSFORM}

\section{A. The principle of image fusion algorithm based on wavelet transforms}

The application of wavelet image fusion principle is the fusion method is applied to the low frequency component and high frequency components of the wavelet decomposition of the original image. In the wavelet transform of an image, the larger the absolute value of wavelet coefficients corresponding to the edge of these more salient features, most of the image fusion algorithm based on wavelet transform to study how to select the wavelet coefficients in the composite image, which is three direction of the high frequency coefficients, so as to achieve the purpose of preserving image edges. Although the choice of the wavelet coefficients (high frequency coefficients) retain the edge of the image features such as a very major role, but 
the scale factor (low-frequency coefficient) determines the contours of the image, the right to choose the scale coefficient is important to improve the visual effects of the synthetic image role.

\section{B. The process of fusion algorithm based on wavelet decomposition}

The algorithm is the wavelet decomposition of the image to get the high-frequency information of the image, as a convicted on the basis of the late goal, the advantage of wavelet transform applied to image fusion image can be decomposed into different frequency domain at different frequency domain the use of different selection rules, the synthetic image multi-resolution decomposition, and thus retain the original image in the composite image and the salient features in different frequency domains.

According to the idea of wavelet image fusion algorithm, the main steps are as follows:

- Geometrically accurate registration for multi-source image;

- Select the appropriate wavelet basis and decomposition level, multi-layer wavelet decomposition, the original image to obtain their approximate coefficients and detail coefficients;

- The fusion rules of the wavelet coefficients according to specific needs, options;

- On the inverse transform of the wavelet coefficients of the fused image.

\section{Image fusion rules}

The image of the low-frequency data to determine the broad contours of the image, and high-frequency data to determine the clarity of the image, the image blurring that its details, the high-frequency information are lost more. Based on the above understanding, the available fusion rules are as follows.

\section{1) Pixel-based fusion rules}

Common pixel-based fusion method is the cross-pixel selection method, selected from the corresponding position of each source image in the wavelet coefficient matrix, the large absolute value of wavelet coefficients as the integration of wavelet coefficients, and then the inverse wavelet transforms fusion images. According to the $\mathrm{LH}, \mathrm{HL}$, and $\mathrm{HH}$ three sub-matrix of the wavelet coefficients of the source image matrix, the absolute value of the corresponding submatrix of wavelet coefficients and the size to determine the fusion matrix of wavelet coefficients $\mathrm{LH}, \mathrm{HL}$, and $\mathrm{HH}$ three sub-matrix (high frequency) wavelet coefficients of the corresponding position in which source image to the corresponding position of the wavelet coefficients.

\section{2) Region-based fusion rules}

Pixel-based fusion rules in the fusion process, on the edge of the high sensitivity of the image preprocessing image strict alignment, otherwise the results will be unsatisfactory, and this increases the difficulty of the pretreatment. Regionbased fusion rules taking into account the correlation between adjacent pixels, reducing the sensitivity to the edge. How to choose the window is the difficulty of this fusion rule, which requires that the wavelet coefficients in the selected window larger, otherwise it will not be able to play the advantage of this method. We can image as constituted by different gray scale region, and the edge of the performance of the objects for the difference of gray. The edge is a very important feature of the images contains valuable information of the target boundary, from the edge of the image location, identification, filtering and other operations. Therefore, we can extract the source of the edge of the map to the edge of the image as a reference around the edge of the integration window $(3 \times 3)$, and then combined with the image information within the region, and used window-based fusion rule fusion, which region-based fusion method.

\section{High-frequency coefficient fusion rules}

For the high-frequency domain, we always want to retain as much as possible details of the input image, and therefore special attention to the striking images in the high frequency. Therefore, the integration of rules and algorithms of the previous wavelet image fusion method, presented here take a Japanese regional mean-variance maximizing new fusion rule based on the coefficient of absolute value and algorithms. Two images A, B fusion, for example, the fusion image of the F. N-layer wavelet decomposition of twodimensional image, the final $(3 \mathrm{~N}+1)$ in different frequency bands, which include $3 \mathrm{~N}$ high frequency band and a low frequency band. The fusion rules and fusion algorithm is:

- Source image A, B, respectively, the N-layer wavelet decomposition;

- Integration of low-frequency part of the image F, take the source image A, B decomposition of weighted average, that is:

$$
C_{N, F}=\left(C_{N, A}+C_{N, B}\right) / 2
$$

Among them, the $C_{N, A}, C_{N, B}$, respectively, to participate in the integration of source images $A$ and $B$ in the low frequency components of wavelet decomposition scale $\mathrm{N}, C_{N, F}$ fusion image $\mathrm{F}$ in the low frequency components of wavelet decomposition scale $\mathrm{N}$.

- The highest decomposition, the wavelet coefficients of high frequency components of the three directions of the compare A, B image, take the absolute value of the wavelet coefficients of the wavelet coefficients of the fused image F.

- In the middle of the decomposition, the local area management pixels (here, take the $3 \times 3$ ) meanvariance image $A$ or $B$, the wavelet coefficients as the fused image $\mathrm{F}$ corresponding wavelet coefficients.

- Determine the wavelet coefficients of the fused image $\mathrm{F}$, the inverse wavelet transform, that is, the fused image $\mathrm{F}$.

\section{E. Low-frequency coefficient fusion rules}

Although the choice of the wavelet coefficients (high frequency coefficients) retain the edge of the image features 
such as a very major role, but the scale factor (low-frequency coefficient) determines the contours of the image, the right to choose the scale coefficient is important to improve the visual effects of the synthetic image role. Choice of the scale coefficient for the low band, there are three options to choose.

The first is the average method, using the mathematical formula is:

$$
C_{N}(Z, P)=0.5 \times C_{N}(X, P)+0.5 \times C_{N}(Y, P)
$$

The average low frequency coefficients directly, without considering the edge of the image, and other features, will reduce the contrast of the image to a certain extent.

The second program is the Burt raised the average method of combining and selection. First, the energy within a small region $\mathrm{Q}$ to represent a significant, if $\mathrm{A}(\mathrm{X}, \mathrm{p})$ is significant that the image $\mathrm{X}$ in the coefficient of $\mathrm{p}$-point scale:

$$
A(X, P)=\sum_{q \in Q} \omega(q) C_{N}^{2}(X, q)
$$

Among them, $\omega$ (q) represents the weight value, the closer from the point $\mathrm{p}$, the greater the weight value. The same can define A (Y, p). Then define the matching matrix R:

$$
R((P))=\frac{2 \sum_{q \in Q} \omega(q) C_{N}(X, q) C_{N}(Y, q)}{A(X, p)+B(X, P)}
$$

Changes in the matching matrix of values between 0 and 1 , close to zero on the two map, it shows a high degree of correlation close to 1 . Matching matrix in a point value is small (less than a certain threshold a) on the choice of a significant high scale coefficients as the scale factor of the composite image; on the choice of the two image scale factor when the value of the matching matrix weighted average of the scale factor at this point as a composite image. Then the integration function can be described as:

$$
C_{N}(Z, P)=W(X, P) \cdot C_{N}(X, P)+W(Y, P) \cdot C_{N}(X, P)
$$

The second program take into account the correlation of the two images, and according to the different correlation were used to select and average method. When the strong correlation of two images, the average method; when the weak correlation of the two images on the choice of the larger point of the local energy. This principle of selection in line with the fact that human eye is more sensitive to the more significant point to a certain extent. So may be inferred that the fused image obtained by such a program would be better than the average fusion image effects. In the second scenario, however, still did not take into account the edge of the image of these significant features, so sometimes it will affect the effect of the fused image.

The third scenario is based on the edge of the options. Image of $\mathrm{X}$ scale factor is defined a variable $\mathrm{E}$ :

$$
E(X, P)=\left(F_{1}^{*} C_{N}\right)^{2}(X, P)+\left(F_{2}^{*} C_{N}\right)^{2}(X, P)+\left(F_{3}^{*} C_{N}\right)^{2}(X, P)
$$

Where * denotes convolution. Similarly, the image Y, define the variable $\mathrm{E}(\mathrm{Y}, \mathrm{p})$. The variable $\mathrm{E}$ reflects to some extent, the edge of the image in horizontal, vertical and diagonal directions. Therefore, in order to retain the details of the original image can be calculated on the scale coefficients of the two images a variable $\mathrm{E}$, and choose $\mathrm{E}$ large scale coefficient as a composite image of the scale factor, so that we can in the fused image retain the original image edge information. Integration of the function expressed as:

$$
C_{N}(Z, P)=W(X, P) \cdot C_{N}(X, P)+W(Y, P) \cdot C_{N}(X, P)
$$

The third option in the pieces of the original image, select the most likely edge points be retained, so you can predict the composite image obtained by this method is relatively clear, the details are more abundant.

\section{EXPERIMENTAL RESULTS AND ANALYSIS}

This paper is based on wavelet transform infrared image and visible image fusion, based on the information contained in the same image source in the visible light and infrared light is different, in order to get the two images useful to us more information than the original image we will both fusion.

Through the comparison of different fusion rules, and finally take the coefficient of low-frequency part of the average, using the coefficient of the high frequency part, whichever is greater of the fusion specifications to ensure maximum retention of information. The expected effect, we want to be able to infrared image with the information contained in the optical image to maximize retained, and fusion in a figure, which contains the information of the infrared and visible light images.

Experiments we have chosen picture named "naviA-t.jpg "," naviB-t.jpg ", as shown in Fig.2, Fig.3.

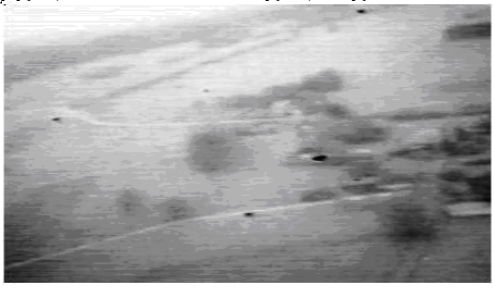

Figure 2. Picture "naviA-t.jpg"

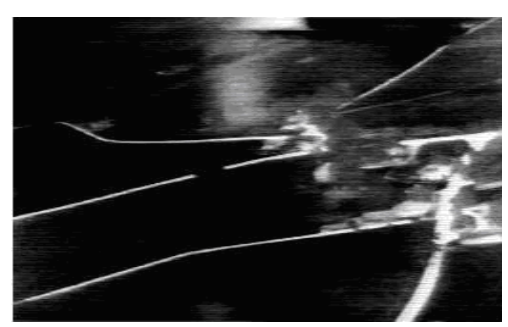

Figure 3. Picture "naviB-t.jpg" 
Picture "naviA-t.jpg" is the ordinary visible light images, pictures naviB-t.jpg and pictures naviA-t.jpg "at the same time in the same image source with the infrared video equipment shooting the picture. Picture "naviB-t.jpg" is the pictures taken with infrared camera equipment.. Two are in the same scene, the infrared image and visible image fusion can concurrently take advantage of the original image information can overcome the limitations and differences exist in terms of geometric, spectral and spatial resolution by a single sensor image, improve the quality of the image, the final image fusion of infrared and visible light image shown in Fig.4.

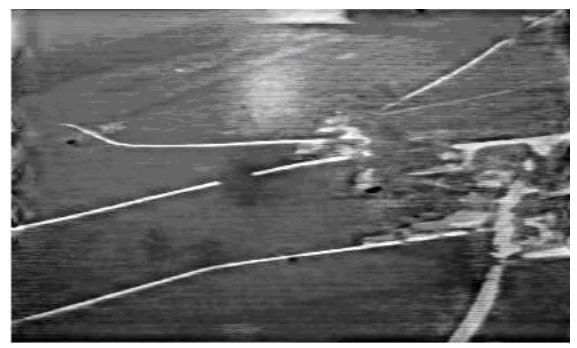

Figure 4. The infrared image and visible image fusion image

We found that through the final fusion image based on wavelet transform infrared image and visible image fusion is successful. Ultimately the infrared image with the information contained in the optical image to maximize the retained and integrated in the way a display, which contains the information of the infrared and visible light images.

\section{CONCLUSION}

Visible image of the spectral information and infrared imagery perspective characteristics of the two image fusion has become inevitable. Therefore, choosing a good fusion algorithm can effectively fused image has the advantages of the two images. The main content of this paper is to explore the image fusion algorithm selected by analyzing the comparison of today's main image fusion algorithm based on wavelet transform image fusion method, will be the integration of image wavelet transform, the image is broken down into different frequency in the frequency domainsection of the decomposition of the transform coefficients, which constitute a series of matrices (subimages), the formation of a wavelet transform pyramid. Experimental results show that the fusion image fusion algorithm is conducive to the overall understanding of the scene, the experimental data and visual effects show that the effectiveness of the algorithm.

\section{ACKNOWLEDGMENT}

This paper supported by Natural Science Foundation of Anhui Province in University（KJ2011B136）, thanks.

\section{REFERENCES}

[1] GEMMA P, "A general framework for multiresolution image fusion:from pixels to regions ,Information Fusion.America,vol.4,pp.295-280,april 2003.

[2] BURT P T, and ADELSON E H, "The Laplacian pyramid as a compact image code,’IEEE Trans Communications.America,vol.31,pp.532-540,april 1983.

[3] CHEN Hao, and WANG Yan-jie, "Study for Image Fusion Based on Wavelet Transform,” MICROELECTRONICS \& COMPUTER.China,vol.27,pp.39-41,may 2010.

[4] Raghavendra R,Dorizzi B,and Rao A, "Particle Swarm Optimization based Fusion of Near Infrared and Visible Images for Improved Face Verfication,"Pattern Recognition.America, vol.44,pp.401411,february 2011

[5] ZHAO Ying-nan,WEN Xue-zhi,and Cheng Ya-ping, "Fase Face Recognition of Sparse Representation Based Fusion of Visible and Near Infrared Images,"Computer Science.China,vol.39,pp.270273,june 2012.

[6] CAO Yi-qin,LEI Zhang-ming,and HUANG Xiao-sheng, "Regionbased algorithm for non-sampling morphologicalwavelet medical image fusion,"Application $\quad$ Research of Computers.China,vol.29,pp.2379-2381,june 2012.

[7] ZHU Ya-hui, and PENG Guo-hua, "Evaluation method of image fusion based on multi-scale PSNR," Application Research of Computers.China,vol.29,pp.2784-2786,july 